\title{
Exploitation of Remote Sensing Data for Land Cover to Habitat Map Translation: A Case Study
}

\author{
Maria ADAMO ${ }^{1}$, Cristina TARANTINO ${ }^{1}$, Vasiliki KOSMIDOU ${ }^{2}$, Zisis PETROU $^{2}$, \\ Ioannis MANAKOS ${ }^{2}$, Valeria TOMASELLI ${ }^{3}$, Richard $\mathrm{LUCAS}^{4}$, \\ Sander MUNCHER ${ }^{5}$ and Palma BLONDA ${ }^{1}$ \\ ${ }^{1}$ CNR-ISSIA, Bari/Italy·adamo@ba.issia.cnr.it \\ ${ }^{2} \mathrm{CERTH}$, Thessaloniki/Greece \\ ${ }^{3} \mathrm{CNR}-\mathrm{IGV}$, Bari/Italy \\ ${ }^{4}$ Aberystwyth University/UK \\ ${ }^{5}$ ALTERRA, Wageningen/Netherlands
}

This contribution was double-blind reviewed as extended abstract.

\begin{abstract}
Focusing on the Food and Agricultural Organization (FAO) Land Cover Classification System (LCCS) and the recently proposed General Habitat Categories (GHCs) classifycation system, this paper illustrates how expert knowledge concerning class spatial arrangement in the scene at hand class, class phenology and class spectral signature in multitemporal EO images can fill the gaps between the two classification systems and provide LC/LU to habitat translation. An application to a Natura 2000 site in Southern Italy which includes a wetland costal area is discussed.
\end{abstract}

\section{Introduction}

The use of Earth Observation (EO) data is a well consolidated approach to provide a synoptic view of land cover/use (LC/LU). Although LC/LU maps are used for indirect mapping of habitats, they are not so clearly relatable to biodiversity in comparison to habitat classifications and provide less scope for monitoring. A conversion from LC/LU classification to habitat classification can be of great utility but differences in definitions and criteria can represent a limitation to the establishment of a unified approach for such translation.

The Food and Agricultural Organisation (FAO) Land Cover Classification System (LCCS) (Di GREGORIO \& JANSEN 2005) was found to be the most useful for translating EO-derived LC/LU to habitat categories (TOMASELLI et al. 2013) since all primarily vegetated LCCS land cover classes are derived from a consistent physiognomic-structural conceptual approach (that combines the classifiers Life Form, Cover and Height) and it allows a better description of natural and semi-natural habitats in comparison to other classification systems. In Europe, natural habitats are usually expressed according to the Annex I of the European Directive (Council Directive 92/43/EEC), the latter being the main European Union legal instrument concerning biodiversity and conservation of natural habitats. 
A recent habitat classification system named General Habitat Categories (GHCs) (BUNCE et al. 2008, 2011) that relies on the concept of Life Forms (LFs) (RAUNKIAER 1934), has been recently developed within recent European projects (i.e., EBONE, BioHab) as a useful approach for monitoring habitats by in-field campaigns and seems suitable also for habitat mapping from remotely sensed data. The mapping of GHCs is based on the identification of Plant Life Forms primarily based on vegetation structure (e.g., trees, shrubs, grass and forbs), phenology, leaf type, vegetation height and additional global, environmental and management qualifiers through a set of expert decision rules (BUNCE et al. 2011).

To date, and within the EBONE project, the expert rules have been used extensively to produce GHC habitat maps for sample areas (typically $1 \mathrm{~km} \times 1 \mathrm{~km}$ ) all over Europe with these supported by in-field campaigns. Similar rules can be applied in order to derive the GHCs directly from a LC/LU maps in FAO-LCCS taxonomy. However, the systematic translation of LCCS maps into GHCs has proven challenging considering the different background of both the taxonomies development, largely because of (KOSMIDOU et al. 2011):

1. The discrepancies between class definitions in the two taxonomies;

2. The different level of detail in the classes definitions;

3. The several one-to-many LCCS to GHC class transitions.

The main purpose of this paper is to provide a generalized automatic framework for translating LCCS maps into GHC maps by using EO data and context sensitive information. Based on the use of different textural features, plants have been separated into two coarse height groups and a GHC map is produced. Such map is then compared with the one also produced through the use of a LIDAR dataset (provided by the Ministero dell'Ambiente, Italy) to evaluate the effectiveness of texture measurements within the LCCS to GHC mapping rule set.

This work was conducted within the three-year BIO_SOS (www.biosos.eu) project, funded within the European Union FP7-SPACE third call, with this aiming to develop a preoperational system for cost effective and timely monitoring of changes in land cover and habitats within and along the borders of protected areas, and judge the effectiveness in protecting and conserving the regions from human impacts. The results discussed hereafter were obtained for one Natura 2000 site in Southern Europe, but the methodology was applied to many European sites within BIO_SOS project.

\section{Input Data and Data Processing}

Expert knowledge is used in this paper to solve the ambiguities in LCCS to GHCs translation by bridging the gap between the two domain-specific knowledge representations. LCCS and GHC classes and their relationships are described in terms of spatial, temporal relations and prior spectral knowledge related to class specific spectral signatures in EO data. The key elements of LCCS to GHC map translation with disambiguation of one-to-many relations include:

- Spatial topological relations. In particular, the adjacency relation was used.

- Temporal relations. To deal with the phenological properties of vegetation, at least 2 images had to be introduced: an image corresponding to the peak of vegetation pro- 
ductivity and an image relative to the post-flush period. Moreover, the two images were used also to deal with properties related to seasonal water coverage.

- Geometric attributes. The requirement of regularity in the shape of a segment was used. This attribute is obtained in eCognition using the Border Index feature (TRIMBLE 2011). Based on a rectangular approximation, this feature describes how jagged a segment is. The smallest rectangle enclosing the image object is created and the Border Index is calculated as the ratio between the perimeter length of the object and the smallest enclosing rectangle.

- Textural Features. First order entropy (occurrence measurements) values are extracted from the green band, which showed the best result in terms of height separability. A kernel window of $3 \times 3$ pixels on the TOA reflectance values resampled in 8 bit was used. Different window sizes were also tested.

- Prior spectral knowledge on class spectral signature. Spectral indices were selected and used as inputs to the translation algorithm, such as the Water Index (WI) and Greenness Index (GI) defined as:

$$
\begin{aligned}
& W I=\rho\left(\lambda_{\text {Blue }}\right) / \rho\left(\lambda_{\text {NIR }}\right), \\
& G I=\rho\left(\lambda_{\text {Red }}\right) / \rho\left(\lambda_{\text {NIR }}\right),
\end{aligned}
$$

where $\rho$ is the TOA value in the bands considered and is calculated as the mean value inside the object.

The study site, Le Cesine (SCI code IT9150032, SPA code IT9150014), belongs to the Natura 2000 network and consists of a variety of habitats including natural, semi-natural and agricultural areas. The retrodunal humid area is one of the most important in Southern Italy and it is formed by two large coastal lagoons and various channels, marshes and wet grasslands.

\section{Results}

In Fig. 1 results of the LCCS to GHC translation using LIDAR data (a) and texture (b) are shown. Additional results of the comparison of the maps obtained by using different texture measures will be discussed during presentation. The findings of this paper confirm the feasibility of translating LC/LU maps into GHC habitat maps based on EO data and expert knowledge. The explicit description of both LCCS classes and GHC classes and their translation (i.e. phenology information) in terms of spatial, both topological and non-topological relations, temporal relations and spectral signatures is at the basis of the automatic mapping of LCCS into GHC habitats. LIDAR data availability allows the identification of the specific GHC class based on height discrimination but texture measures can help to discriminate two macro height classes, when LIDAR data are not available. The methodology can be applied to pre-existing validated Land Cover map produced in FAO-LCCS taxonomy or translated in such taxonomy, by taking into account the errors related to such translation. This means that GHC maps can be produced also in the past and used for habitat change detection if archive EO multi-temporal images (two seasons) close to the date of the LC map production and validation are available on the area. 

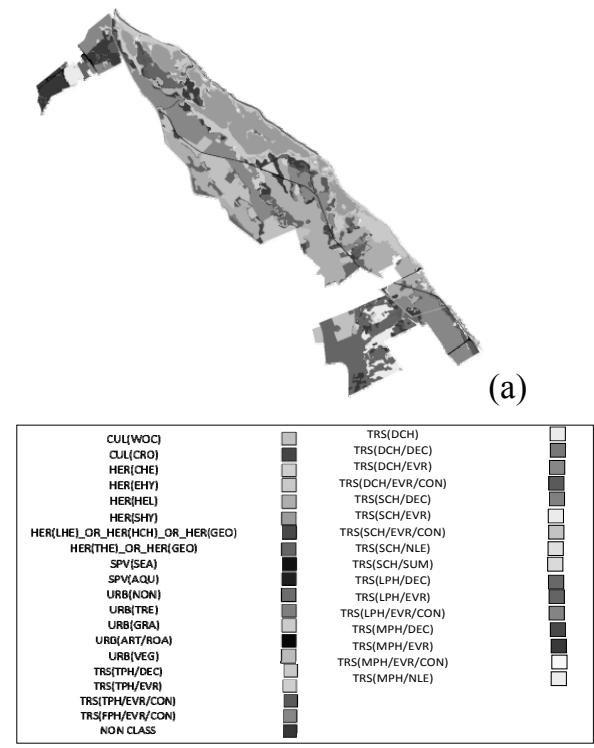

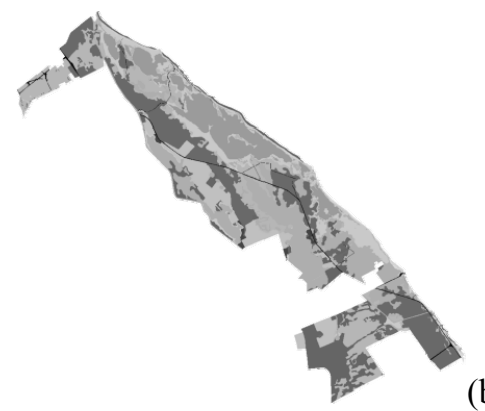

(b)

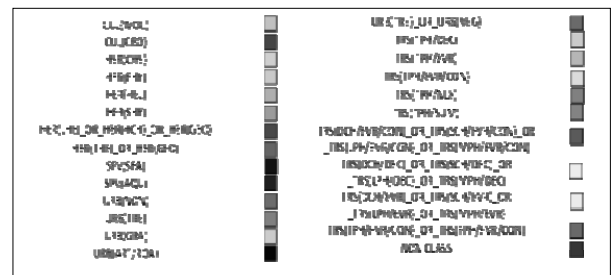

Fig. 1: GHC map produced for Le Cesine site using LIDAR data (a) and texture (b)

\section{References}

Bunce, R. G. H., Bogers, M. M. B., Roche, P., WalczaK, M., GeiJzendorffer, I. R. \& Jongman, R. H. G. (2011), Manual for Habitat Surveillance and Monitoring and Vegetation in Temperate, Mediterranean and desert Biomes. Alterra-EBONE_Handbook v20110131.

Bunce, R. G. H., Metzger, M. J., Jongman, R. H. G., Brandt, J., De Blust, G., ElenaRossello, R., Groom, G. B. et al. (2008), A standardized procedure for surveillance and monitoring European habitats and provision of spatial data. Landscape Ecology, 23, $11-25$.

Di Gregorio, A. \& Jansen, L. J. M. (2005), Land Cover Classification System (LCCS): classification concepts and user manual. Software version 2. Food and Agriculture Organization of the United Nations, Rome.

Kosmidou, V., Petrou, Z., lucas R. M., Tomaselli, V., Petrou, M., Bunce, R., Bogers, M., Mücher, A., Tarantino, C., Blonda, P. \& Baraldi, A. (2011), Software for habitat maps production fro LC. BIO_SOS Biodiversity Multisource Monitoring System: from Space TO Species 868 (BIO_SOS) Deliverable D6.10. http://www.biosos.wur.nl/NR/rdonlyres/935FA85D-DC21-4FDC-

ADE6037FC172A312/156409/BIO_SOS_D610_software_for_habitat_maps_from_LC map.pdf.

RAUNKIAER, C. (1934), The life forms of plants and statistical plant geography, being the collected papers of C. Raunkiaer. Oxford, Clarendon. 
Tomaselli, V., Dimopoulos, P., Marangi, C., Kallimains, A. S., Adamo, M., Tarantino, C., Panitsa, M., Terzi, M., Veronitco, G., Lovergine, F., Nagendra, H., LUCAS, R., MAirota, P., MÜCher, S. \& BlondA P. (2013), Translating Land cover/Land use Classifications to Habitat Taxonomies for Landscape Monitoring: A Mediterranean Assessment, Landscape Ecology. DOI 10.1007/s10980-013-9863-3.

TRIMBLE (2011), Ecognition Developer 8.7. Reference Book.

http://www.ecognition.com/. 